\title{
You may (not always) experience what you expect: In search for the limits of the placebo and nocebo effect
}

A wealth of research shows that the expectancy to improve, either by verbal suggestion or by learning experiences, results in less pain and suffering [8]. There are also intriguing studies showing the relevance and usefulness of placebo in clinical settings [4]. Far less research is available on the reverse, the nocebo effect. Overall, results of these studies are concordant with the placebo research: expectation of adverse somatosensory events may induce their experience. It is acknowledged that the effects of placebo and nocebo are genuine, not artefacts of methodological shortcomings. It is also clear that the mechanisms underlying these phenomena are not unitary, but vary as a function of contextual and individual specificities. Although the key mediator is expectancy, different physiological and psychological mechanisms likely contribute under different circumstances.

In the current issue of Pain, Van Laarhoven and colleagues [10] investigated effects of nocebo and placebo instructions on pain and itch. Healthy volunteers received verbal instructions that only slightly varied as to the somatosensory events to be experienced. The overall results are very clear. We tend to experience what we expect. More pain is experienced when participants were informed that most subjects experienced the stimuli as painful. More itch is experienced when participants were informed that most subjects experienced the stimuli as itch. Likewise, suggestions that the intensity of the sensations will decrease results in a decrease of the experience. This elegant study adds to the vast database on placebo and nocebo effects, however, the paper also reveals some remarkable novel findings. First, the paper focuses on the effects of verbal suggestions on pain and itch. Intriguingly, itch seemed to be more malleable to verbal suggestion than pain. Second, the authors used a wide range of somatosensory stimuli including mechanical stimuli (monofilaments), a series of short electrocutaneous stimulus at fixed intensity, an electrocuteanous stimulus at tolerance level, and the application of histamine. Apparently not all stimuli are equally potent in inducing a placebo or nocebo effect. All but mechanical stimuli successfully induced pain following nocebo instructions. These findings are subtle at first examination, but they raise a fundamental question that deserves scrutiny: Where are the limits of the placebo and nocebo effect?

Taken together, the literature on placebo and nocebo seems to indicate that we experience what we expect. Although true to some extent in some situations, we do not live in a hallucinatory world, where expectations are self-fulfilling prophecies and construct our reality. There are constraints. Most often we readily detect when reality does not meet our expectations. Indeed, a key feature of successful adaptation is the ability to be sensitive to a lack of confirmation of our expectations. Such an error detecting mechanism is at the foundation of cognitive theories of learning and extinction [9], and has recently gained considerable interest in cognitive neuroscience (for discussion see [6]). In this view, incoming sensory information is compared to a template that reflects the expectation about forthcoming events. When expected and observed information diverge, a neural 'prediction error' signal is generated, which in turn leads to updating of expectations. In line with this view, studies in low back pain patients indicate that pain expectancies do not induce (more) pain, but rather generate a rapid adaptation of the pain expectation [2]. The same mechanism is also key in many cognitivebehavioural techniques. For example, during behavioural exposure patients with musculoskeletal pain are asked to perform those movements they fear the most. The recognition that expected catastrophes, in fact, do not occur is the quintessential event for behavioural change and therapeutic success $[3,5]$. While preparing patients for painful, medical procedures, it is common practice to inform correctly about these experiences. Presenting the to 
be experienced event as worse may create unnecessary anticipatory anxiety, and even avoidance. Conversely, presenting the experience as less painful may contribute to more pain (see [1]), and may leave patients distrusting their health care providers.

It is clear that two opposing mechanisms are at play: Expectations can bias perception but likewise, (disconfirming) sensory evidence can result in updating of expectations. A great challenge is to search for the balance between these opposing mechanisms, and to identify each mechanism's boundaries. Here, we propose some ideas that allow a rigorous experimental approach. (1) Somatosensory experiences that are ambiguous are more malleable to expectations than experiences with clear perceptual characteristics. (2) Expectations about experiences that remain within a certain margin of the actual experience/stimulus will be more susceptible to placebo and nocebo effects, otherwise the expectations will lead to disconfirmations of expectations. (3) Expectations are less powerful when they are associated with behavioural costs (see [7]). We therefore may expect an asymmetry between placebo and nocebo effects. (4) The effects of expectancies may vary as a function of repeated experiences.

\section{Conflict of interest statement}

The authors have no conflict of interest in relation to this commentary.

\section{References}

1. Crombez G, Baeyens F, Eelen P. Sensory and temporal information about impending pain: The influence of predictability on pain. Behav Res Ther 1994;32:611-622.

2. Crombez G, Vervaet L, Lysens R, Eelen P, Baeyens, F. Do pain expectancies cause pain in chronic low back patients? A clinical investigation. Behav Res Ther 1996;34:919925.

3. Foa EB, Kozak MJ. Emotional processing of fear: Exposure to corrective information. Psychol Bull 1986;99: 20-35.

4. Goossens M E, Vlaeyen JW, Hidding A, Kole-Snijders A, Evers SM. Treatment Expectancy Affects the Outcome of Cognitive-Behavioral Interventions in Chronic Pain. Clin J Pain 2005;21:18-26.

5. Leeuw M, Goossens MEJB, Linton SJ, Crombez G, Boersma K, Vlaeyen JWS. The fearavoidance model of musculoskeletal pain: current state of scientific evidence. Behav Med 2007;30: 77-94.

6. Niv Y, Schoenbaum G. Dialogues on prediction errors. Trends Cogn Sci 2008;12:265272.

7. Poulos CX, Cappell H. Homeostatic theory of drug tolerance: A general model of physiological adaptation. Psychol Rev 1991;98:390-408.

8. Price DD, Finniss, DG, Benedetti F. A comprehensive review of the placebo effect: Recent advances and current thought. Annu Rev Psychol 2008;59: 565-590. 
9. Rescorla RA \& Wagner AR. A theory of Pavlovian conditioning: Variations in the effectiveness of reinforcement and nonreinforcement. In A Black \& WF Prokasy (Eds). Classical conditioning II, New York: Appleton-Century-Crofts. 1972

10. Van Laarhoven (reference of paper in PAIN)

Geert Crombez

Department of Experimental-Clinical and Health Psychology, Henri Dunantlaan 2, B-9000 Gent, Belgium. E-mail address: Geert.Crombez@UGent.be

Katia Wiech

Nuffield Department of Clinical Neurosciences (Nuffield Division of Anaesthetics), FMRIB Centre University of Oxford John Radcliffe Hospital Oxford, OX3 9DU England UK, and Research Group Health Psychology, University of Leuven, B-3000 Leuven, Belgium. 